\title{
Targeting the Nuclear Receptor LRH-1 with Synthetic Agonists
}

\author{
Suzanne G. Mays ${ }^{1}$, C. Denise Okafor ${ }^{1}$, Richard Whitby ${ }^{3}$, Devrishi Goswami ${ }^{4}$, Józef \\ Stec $^{3}$, Autumn Flynn ${ }^{2}$, Michael Dugan ${ }^{2}$, Nathan Jui ${ }^{2}$, Patrick Griffin ${ }^{4}$, and Eric A. Ortlund ${ }^{1}$ \\ ${ }^{1}$ Department of Biochemistry, Emory University School of Medicine, and the \\ ${ }^{2}$ Department of Chemistry, Emory University, Atlanta, GA 30322; ${ }^{3}$ School of Chemistry, \\ University of Southampton, Southampton, United Kingdom; and the ${ }^{4}$ Scripps Research \\ Institute, Jupiter, FL.
}

\begin{abstract}
Liver receptor homolog 1 (NR5A2, LRH-1) is an orphan nuclear hormone receptor that regulates diverse biological processes, including lipid and glucose metabolism, proliferation, and the resolution of endoplasmic reticulum stress. Preclinical studies have demonstrated a great therapeutic potential of targeting $\mathrm{LRH}-1$ for treatment of metabolic diseases, such as diabetes; however, development of LRH-1 modulators has been challenging. In a recent study, systematic modifications to one of the few known chemical scaffolds capable of activating LRH-1 failed to improve efficacy substantially. Moreover, mechanisms through which LRH-1 is activated by synthetic ligands are entirely unknown. Here, we use x-ray crystallography, molecular dynamics simulations, and cellular activation assays to explore conformational changes and receptor-ligand interactions associated with $\mathrm{LRH}-1$ activation by a set of related agonists. Unlike phospholipid (PL) LRH-1 ligands, these agonists bind deep in the pocket and do not interact with residues near the mouth, nor do they expand the pocket like PLs. Unexpectedly, two closely related agonists with similar efficacies (GSK8470 and RJW100) exhibit completely different binding modes. The dramatic repositioning is influenced by a differential ability to establish stable, face-to-face $\pi$ - $\pi$-stacking with LRH1 residue $\mathrm{H} 390$, as well as by a novel polar interaction mediated by the RJW100 hydroxyl group. The differing binding modes result in distinct mechanisms of action for the two agonists. This work reveals a previously unappreciated complexity associated with $\mathrm{LRH}-1$ agonist development and offers insights into rational design strategies.
\end{abstract}

